1117

\section{POST BLOOD OR MARROW TRANSPLANT COMPLICATIONS IN A COHORT OF CHILDREN WITH RELAPSED/REFRACTORY A.L.L. OR A.M.L: A SINGLE INSTITUTION STUDY}

\author{
R. Rohrer \\ Epidemiology, University of Pittsburgh, \\ Pittsburgh, PA, USA
}

Background and aims: This study examines the types of postblood or marrow transplant complications in patients treated at the Children's Hospital of Pittsburgh in a five year period (2004-2009). The study will focus on complications diagnosed in the 0-100 day time span while children are frequently hospitalized and will compare type and rate of complications between children diagnosed with acute lymphoblastic leukemia and acute myelongenous leukemia.

Methods: The author will examine patient records and interview treatment teams and patient family on the severity and length of complications. The author has developed separate forms for these sets..

Results: Initial results show that patients with A.M.L. who received greater transplant conditioning had more severe complications particularly graft versus host disease. The study will continue tol examine each variable such as age, remission status and overall health status pre transplant in order to best assess the final data.

Conclusions: Blood and marrow transplants although life saving remain extremely toxic and post-transplant complications are common. The development of a less toxic "mini transplant" at the Children's Hospital of Pittsburgh addresses these issues however further studies such as those conducted by the Children's Oncology Group are needed to fine tune risk factors prior transplant and assess the greater susceptibility of some patients to complications.

1118

INTRODUCTION FOR NEW STAFF, REGISTERED NURSES, AT THE PEDIATRIC INTENSIVE CARE UNIT - PICU, AT SKANE UNIVERSITY HOSPITAL, LUND, SWEDEN

\section{K. Pappila}

PICU, Skane University Hospital, Lund, Sweden

Aim: The introduction program will give the new employee (registered nurse, RN) a uniform introduction and educational level regardless the mentors. The introduction program will support both mentors and the new employee.

Method: The staff management will allow time for practical and theoretical introduction as required individually. The RN, will have two mentors, experienced registered nurses, and one assistant nurse. For the first five weeks the RN will follow these mentors schedule full time. The RN will work side by side with the mentor. After 10 weeks the intensive introduction is over. Included in the Introduction program is: Checklist, everything that need review at PICU. Theoretical compendia, including routines and facts on how to work at PICU. Education during three days in the first year. Specialist nurses arrange and teach during these opportunities. The RN, the mentors and the teamleader meet after 5 and 10 weeks, 6 and 12 months for follow up.

Result: At the end of the first five weeks the RN is able to handle commonly occurring patients as well as being familiar with PICUs routines and guidelines with some guidance from the mentors. After approximately 10 weeks the RN can independently handle the above mentioned category of patients. She has the liability but have her mentor working in the same room.

Conclusion: The introduction program give opportunity for new employees to prepare for their new tasks no matter the mentors or their own background. The RN get a stable frame to lean on to when new.

\section{9}

\section{TRAINING OF NURSES AND DOCTORS AT CHILDREN'S HOSPITAL IN ASSESSMENT AND TREATMENT OF ACUTELY ILL CHILDREN}

\author{
L. Hansson \\ Childrens Hospital in Lund, Skane University \\ Hospital, Lund, Sweden
}

Aim: The Children's Hospital in Lund has an objective to increase the competence in assessment and treatment of acutely ill children.

Method: The child is first assessed by nurses and doctors on each ward. If they in the assessment of vital signs find abnormal values a team with an intensivist and a nurse from the PICU will be called. Together with the responsible doctor and nurse, the team together will make a second assessment of the child. The PICU team can only recommend 